\title{
Linguagem e memória autobiográfica de adolescentes usuários de drogas
}

\section{Language and autobiographical memory of adolescent drug users}

\author{
Christian César Cândido de Oliveira ${ }^{1}$, Cláudia Scheuer $^{2}$, Sandra Scivoletto ${ }^{3}$
}

\begin{abstract}
RESUMO
Objetivo: Resgatar, por meio da linguagem, a memória autobiográfica de adolescentes usuários de drogas/álcool. Métodos: Participaram do estudo 25 adolescentes usuários e 25 não usuários de álcool/drogas com idades entre 13 e 17 anos, do sexo masculino. Após coleta da linguagem oral, a análise deste material foi baseada no modelo que privilegia a estrutura discursiva em cenário, complicação, resposta interna, tentativa, conseqüência e reação. Resultados: O grupo de usuários produziu menos tentativa $(\mathrm{p}=0,010)$, conseqüência $(\mathrm{p}=0,030)$ e reação $(\mathrm{p}=0,023)$, indicando diferente perfil na linguagem de ambos os grupos. Conclusão: O perfil da memória autobiográfica de adolescentes usuários de drogas é diferente do de adolescentes não usuários. Este dado pode estar relacionado ao comprometimento no funcionamento cognitivo e/ou de linguagem, assim como de aspectos sociais e psíquicos, ocasionando interrupções na seqüência da lembrança.
\end{abstract}

Descritores: Memória; Linguagem; Neuropsicologia; Transtornos relacionados ao uso de substâncias; Adolescente

\section{INTRODUÇÃO}

O uso de drogas tem maior impacto em adolescentes do que em adultos, porque, apesar de menor tempo de consumo, os jovens estão em desenvolvimento. Nesta fase, as con-

(1) Membro da equipe do Ambulatório de Adolescentes e Drogas do Serviço de Psiquiatria da Infância e Adolescência do Hospital das Clínicas da Faculdade de Medicina da Universidade de São Paulo - USP - São Paulo (SP), Brasil; Pós-graduando do Programa de Pós-Graduação Stricto Senso em Ciências da Reabilitação da Faculdade de Medicina da Universidade de São Paulo - FMUSP - São Paulo (SP), Brasil.

(2) Doutora em Lingüística pela Faculdade de Filosofia, Letras e Ciências Humanas da Universidade de São Paulo - USP - São Paulo (SP), Brasil; Docente do Programa de Pós-graduação em Ciências da Reabilitação do Departamento de Fisioterapia, Fonoaudiologia e Terapia Ocupacional da Faculdade de Medicina da Universidade de São Paulo - USP - São Paulo (SP), Brasil.

(3) Doutora em Psiquiatria pela Faculdade de Medicina da Universidade de São Paulo - FMUSP - São Paulo (SP), Brasil; Coordenadora do Grupo Interdisciplinar de Estudos de Álcool e Drogas do Instituto de Psiquiatria do Hospital das Clínicas da Faculdade de Medicina da Universidade de São Paulo - USP - São Paulo (SP), Brasil; Chefe do Ambulatório de Adolescentes e Drogas do Serviço de Psiquiatria da Infância e Adolescência do Hospital das Clínicas da Faculdade de Medicina da Universidade de São Paulo - USP São Paulo (SP), Brasil; Docente dos programas de Pós-Graduação em Psiquiatria e Fisiopatologia Experimental da Faculdade de Medicina da Universidade de São Paulo - USP - São Paulo (SP), Brasil.

Trabalho realizado no Ambulatório de Adolescentes e Drogas do Grupo Interdisciplinar de Estudos de Álcool e Drogas do Serviço de Psiquiatria da Infância e Adolescência do Instituto e Departamento de Psiquiatria do Hospital das Clínicas da Faculdade de Medicina da Universidade de São Paulo - HCFMUSP - São Paulo (SP), Brasil.

Endereço para correspondência: Christian César Cândido de Oliveira. Av. Jabaquara, 1469/112, B1. A3, Mirandópolis, São Paulo - SP, CEP 04045002. E-mail: christianfonoaudio@yahoo.com.br

Recebido em: 23/2/2007; Aceito em: 29/5/2007 seqüências do uso são observadas nas áreas escolar, legal, familiar e física ${ }^{(1)}$.

Entre os sintomas físicos, as alterações cognitivas têm grande relevância, visto que as drogas podem alterar o funcionamento do cérebro do adolescente, período da vida marcado por importantes mudanças psicológicas e cognitivas ${ }^{(2-3)}$. A literatura relata as possíveis consequiências decorrentes do uso de drogas, tais como déficit de atenção ${ }^{(4)}$, dificuldades na aprendizagem ${ }^{(5)}$, rupturas na seqüência do pensamento ${ }^{(6)}$ e prejuízo na memória ${ }^{(7)}$.

Estudos apontam que usuários de drogas podem ter alterações na memória autobiográfica ${ }^{(8-9)}$, um tipo de memória que permite ao sujeito registrar e evocar informações num determinado contexto espacial e temporal, por vezes inespecíficos, permitindo que ele se recorde de fatos de sua história pessoal, familiar ou social ${ }^{(10)}$.

Apesar de a memória autobiográfica desenvolver-se desde o nascimento e acompanhar o indivíduo durante toda a sua vida, é na adolescência que o sujeito passa a relacionar eventos previamente vivenciados iniciando um processo de construção de uma história de vida pessoal de maneira coerente e consciente ${ }^{(11)}$.

A memória autobiográfica pode ser acessada por meio de diversos estímulos, como música, imagens, fotos ou faces, questionários padronizados ou discurso oral livre; porém, a expressão da evocação mais relatada na literatura é a verbal, mostrando forte relação entre a memória e a linguagem $^{(12)}$.

Alguns pesquisadores sugerem que as memórias autobiográficas têm caráter de narrativa porque uma pessoa organiza a experiência, fato ou acontecimentos de sua vida, similarmente à organização de uma história ${ }^{(13)}$. 
A linguagem oral, a mais elaborada e a mais simbólica capacidade cognitiva do ser humano, representa, verifica e conhece como se organizam os eventos, fatos e experiências de toda e qualquer natureza humana ${ }^{(14)}$. Por meio da linguagem, a memória autobiográfica pode ser analisada de forma objetiva por meio das sequiências de ações e eventos, ou subjetiva, na relação entre o recordar e inferir ${ }^{(13)}$.

Quando uma pessoa fala sobre um evento autobiográfico específico, geralmente inclui elementos considerados pistas facilitadoras para o acesso à recordação global. Dentre estes elementos, destacam-se as informações temporais, localização espacial e, ainda, aquelas relacionadas a outras pessoas presentes no momento da construção da lembrança ${ }^{(15)}$. Juntos, estes elementos auxiliam no estabelecimento de um padrão estável de ativação da memória que, conseqüentemente, é verbalizada com maior clareza e veracidade.

Estes elementos estão estruturados na linguagem oral e podem ser identificados utilizando modelos teóricos que privilegiam a estrutura desta linguagem ${ }^{(16)}$ : cenário, complicação, resposta interna, tentativa, conseqüência e reação.

É na linguagem e por meio dela que se podem obter informações sobre a percepção que uma pessoa tem sobre sua própria história de vida ${ }^{(17)}$ e das relações desta história com a de outras pessoas em diferentes contextos e sentidos.

Apesar de existirem muitas informações a respeito da memória autobiográfica e de sua relação com a linguagem, não foram encontrados, na literatura nacional e internacional consultada, estudos com adolescentes usuários de drogas.

Desta forma, o objetivo deste estudo foi analisar, por meio da linguagem, a memória autobiográfica de adolescentes usuários e não usuários, utilizando o modelo de linguagem de Peterson e McCabe ${ }^{(16)}$.

Analisando-se a linguagem contida na memória autobiográfica de adolescentes usuários de drogas, se pode obter importantes informações sobre o funcionamento lingüístico e sobre a integração entre a cognição e a emoção, áreas geralmente prejudicadas em tais sujeitos. Assim, este estudo torna-se importante por possibilitar ao fonoaudiólogo um novo campo de atuação clínica, além de fornecer à equipe interdisciplinar informações que permitam melhor compreensão deste transtorno.

\section{MÉTODOS}

\section{Desenho do estudo}

Foi realizado um estudo observacional, transversal e comparativo autorizado pela Comissão de Ética para Análise de Projetos de Pesquisa (CAPPesq, $n^{\circ}$. 636/03) do Hospital das Clínicas da Faculdade de Medicina da Universidade de São Paulo (HC-FMUSP).

\section{Amostras}

A população desta pesquisa foi composta por 50 indivíduos, dividida em duas amostras: 25 adolescentes usuários e 25 adolescentes não usuários de drogas/álcool.
Amostra de usuários de drogas

Foram avaliados indivíduos em tratamento no Ambulatório de Adolescentes e Drogas do Serviço de Psiquiatria da Infância e Adolescência (SEPIA) do Instituto de Psiquiatria (IPq) do Hospital das Clínicas da Faculdade de Medicina da Universidade de São Paulo (HC-FMUSP).

Foram selecionados sujeitos do gênero masculino com idades entre 11 e 17 anos e 11 meses e diagnóstico de abuso ou dependência de álcool e/ou drogas, de acordo com o DSMIV ${ }^{(18)}$, sem qualquer patologia clínica e/ou psiquiátrica (comprometimento orgânico ou psiquiátrico moderado ou grave) que necessitasse de internação em hospital geral ou psiquiátrico para tratamento e investigação diagnóstica.

A faixa etária dos sujeitos deste estudo foi pré-determinada pelo Ambulatório onde os dados foram coletados. Tratando-se de um estudo comparativo, a amostra de não usuários foi pareada com a de usuários, respeitando a mesma faixa.

\section{Amostra de não usuários de drogas}

Esta amostra foi obtida em uma Escola Municipal de Ensino Fundamental da Cidade de São Paulo.

Foram selecionados sujeitos do gênero masculino com idades entre 11 e 17 anos e 11 meses, sem qualquer patologia clínica e/ou psiquiátrica (comprometimento orgânico ou psiquiátrico moderado ou grave) que necessitasse de internação em hospital geral ou psiquiátrico para tratamento e investigação diagnóstica e que não fizessem uso de álcool, droga ou medicamentos tais como benzodiazepínicos ou neurolépticos.

\section{Características das amostras}

Para caracterizar as amostras foram aplicados: Protocolo para a coleta de informações sócio-demográficas e relativas ao uso de drogas (extraído e modificado do Ambulatório de Adolescentes e Drogas do HC-SEPIA-IPq-HC-FMUSP); Entrevista clinica; Escala da Associação Brasileira dos Institutos de Pesquisa de Mercado ${ }^{(19)}$ e Questionário para detectar uso, abuso ou a dependência de drogas nos sujeitos da amostra de não usuários ${ }^{(20-22)}$.

Ambas as amostras são equivalentes em relação a nível socioeconômico (Classe $\mathrm{C}$ - a mais incidente), idade (usuários, média de 15,48 anos e não usuários, média de 15,16 anos $-\mathrm{p}=0,251)$ e nível escolar (52\% dos usuários e $60 \%$ dos não usuários cursaram ou estavam cursando o ensino médio - $\mathrm{p}=0,7757$ ); além de ambas as amostras pertencerem a escolas da rede pública do Estado de São Paulo.

As drogas mais utilizadas na amostra de usuários estão descritas no Quadro 1.

\section{Procedimentos para a coleta da linguagem e para a análise da memória autobiográfica}

Para acessar os conteúdos implicados e contidos na memória autobiográfica por meio da linguagem oral, foram obtidas informações sobre ALGUM evento ocorrido na infância por meio uma pergunta aberta: "Conte-me uma história que tenha acontecido com você em sua infância". Não 
Quadro 1. Drogas mais utilizadas na amostra de usuários

\begin{tabular}{|lcc|}
\hline Droga & $\begin{array}{c}\text { Percentual de } \\
\text { usuários }\end{array}$ & $\begin{array}{c}\text { Idade do } \\
\text { primeiro uso (média) }\end{array}$ \\
\hline Tabaco & $92 \%$ & 12,17 \\
Álcool & $88 \%$ & 11,36 \\
Maconha & $92 \%$ & 12,91 \\
Cocaína inalada & $36 \%$ & 14,11 \\
Cocaína fumada - crack & $16 \%$ & 13 \\
Benzodiazepínicos ou barbitúricos & $4 \%$ & 11 \\
Inalantes & $8 \%$ & 14,5 \\
Alucinógenos & $8 \%$ & 14 \\
\hline
\end{tabular}

houve qualquer interferência do avaliador nas produções orais, mesmo nas de pequena extensão.

A linguagem contida nos relatos foi transcrita literalmente em português standard falado no Brasil e, as lembranças, analisadas de acordo com o modelo de Peterson e McCabe ${ }^{(16)}$, que preconiza a identificação de elementos capazes de desencadear a seqüência lógica da organização da linguagem oral ou escrita, quando narrada ou relatada. Para esses autores, tais elementos são: cenário, complicação, resposta interna, tentativa, conseqüência e reação.

O cenário trata da caracterização física e/ou psíquica inicial dos personagens e do ambiente, trazendo dados de localização espaço-temporal; a complicação reflete o(s) evento(s) que o sujeito procura resolver, equivalendo à ação e ao principal motivo que o levou à recordação; a resposta interna evidencia a mobilização do personagem a partir de uma mudança interna, os sentimentos envolvidos com a apresentação da complicação e a sua impressão pessoal; a tentativa reflete a ação que é direcionada ao problema alvo com o intuito de resolvê-lo; a conseqüência é o reflexo da tentativa, podendo ser o sucesso ou o fracasso da resolução; e a reação elucida o final da estória e os sentimentos envolvidos com a lembrança.
Foi atribuído 1 (um) ponto para elementos presentes, e 0 (zero) ponto quando ausentes; a pontuação máxima total poderia ser seis pontos e a mínima zero. A estatística aplicada ao estudo foi o teste Qui-Quadrado, com nível de significância 0.05 .

Nas Tabelas 1 e 2 são descritas duas transcrições e as respectivas análises da linguagem, de acordo com Peterson e McCabe $^{(16)}$

\section{Transcrição 1}

"Foi quandu é, meu pai é é bebia é, i chegava todo dia bêbadu i ia batê na minha mãe, aí eu eu tinha uns novi anu tá ligadu, aí eu comecei pá a pegá raiva tá ligadu, aí eli ia pra cima da minha mãe, eu era piquinininhu i ia pra cima deli, aí uma veiz eli quasi mi jogô pra baixu, aí minha casa é tipu um muru altu i baixu é uma oficina i quasi eli mi jogô pra baixu, aí minha mãe feiz B.O. tudu aí num deu im nada pra eli, aí pá fui crescenu, se tá ligadu, i eli foi continuanu batenu na minha mãe, aí quandu eu tinha meus onzi anu eli foi batê na minha mãe, aí nu mi sigurei mais não, tudu, aí logo peguei eli, tá ligadu, aí foi assim manu, começamu a tretá todu dia, todia nóis brigava aí mãe começô a cansá i mandô eli imbora, aí é melhor eli lá longi lá qui nóis tá tá si danu du qui eli aí im casa aí eli briganu cá minha mãe i fazenu ela sofrê".

\section{Transcrição 2}

"Se sabe qui adoro falar di quandu era piquenu purque eu tinha muitu primu i nóis brincava muitu. Tevi um dia qui eu tava num muru i eu caí purque minha prima Joana mi impurrou, tava brincandu di pega pega, aí eu quebrei minha perna só qui foi mó legal purque todo mundu ficou mi mimandu i assinandu u nomi nu gessu".

Tabela 1. Análise da linguagem ${ }^{(16)}$ : transcrição 1

\begin{tabular}{llc}
\hline Elementos & Descrição & Pontuação obtida \\
\hline Cenário & Pai alcoolizado, mãe agredida, filho agredido. & 1 \\
Complicação & Dar fim à tortura da mãe. & 1 \\
Resposta Interna & Começa a "pegar raiva". & 1 \\
Tentativa & Espera o tempo para crescer e tentar fazer algo. & 1 \\
Conseqüência & Enfrenta o pai, as brigas se tornam diárias e a mãe se separa. & 1 \\
Reação & Aparentemente as coisas melhoram porque a mãe pára de sofrer e cada um passa a viver sua vida. & 1 \\
\hline Total & & 6 \\
\hline
\end{tabular}

Tabela 2. Análise da linguagem(16): transcrição 2

\begin{tabular}{llc}
\hline Elementos & Descrição & Pontuação obtida \\
\hline Cenário & Sujeito, prima, muro & 1 \\
Complicação & Brincar de pega-pega & 1 \\
Resposta Interna & Adora a infância porque gostava de brincar com os muitos primos & 1 \\
Tentativa & Subir no muro para brincar & 1 \\
Conseqüência & Cai e quebra a perna & 1 \\
Reação & Foi uma experiência boa, porque todos o mimaram e assinaram os nomes no gesso & 1 \\
\hline Total & & 6 \\
\hline
\end{tabular}


Com o objetivo de garantir a fidedignidade do estudo, após o pesquisador ter analisado as 50 produções orais, foram treinados dois juizes, fonoaudiólogos que, analisando parte destas produções (10\% da população: três usuários e três não usuários), de acordo com procedimento que assegura confiabilidade da pesquisa ${ }^{(23)}$, utilizaram o modelo teórico preconizado para este trabalho.

O teste estatístico Kappa mostrou forte concordância entre o pesquisador e os juizes $(\mathrm{k}<0,001)$.

\section{RESULTADOS}

A partir das análises, observou-se que não houve diferença entre as amostras de usuários e não usuários para os itens: cenário, complicação e resposta interna. Porém, houve diferença significativa para os elementos: tentativa, conseqüência e reação itens finais menos incidentes na estrutura da linguagem de usuários, refletindo que, para estes sujeitos, a lembrança é menos detalhada e elaborada. A Tabela 3 descreve o percentual de presença e ausência destes itens.

\section{DISCUSSÃO}

O desenvolvimento psíquico e da linguagem permite que, na adolescência, as lembranças sejam organizadas e analisadas pelo próprio sujeito, de maneira mais complexa ${ }^{(2,24)}$. É esta a fase da busca de uma identidade psicosocial, além de ser o período em que o individuo deve conseguir raciocinar hipoteticamente sobre sua vida, relacionando o passado (eventos vividos) e o futuro (eventos planejados), ao presente ${ }^{(25)}$. Assim, a identificação de elementos lingüísticos contidos na recordação, torna-se de fundamental relevância.

Muitos estudos a respeito da organização da memória autobiográfica têm examinado a dinâmica destes elementos presentes na verbalização do acontecimento autobiográfico. Mais do que capazes ordenar o evento, estes elementos facilitam a recuperação, de forma que um interlocutor consiga visualizar o fato, sem ter participado da construção da memória. Tais elementos permitem um discurso com maior clareza de idéias, riqueza de detalhes e informações imprescindíveis para a compreensão do fato vivenciado ${ }^{(26)}$.

Os dados encontrados neste estudo mostram que alguns elementos na estrutura da linguagem representativa da memória autobiográfica são igualmente recordados por usuários e não usuários. Ambas as amostras caracterizam o ambiente e os personagens (cenário), relatam o problema que motivou a recordação (complicação), e demonstram os sentimentos envolvidos com a apresentação deste problema (resposta interna).

Tais dados permitem inferir que há uma seqüência temporal e coerente dos fatos recordados por todos os participantes da pesquisa; porém, na amostra de usuários, parece haver uma interrupção nesta sequiência, já que os outros três elementos (tentativa, conseqüência e reação), são significativamente omitidos por estes sujeitos. Os dados apontam que usuários não lembram ou lembram menos sobre questões de suas vidas.

Nesta óptica, o uso de drogas parece interferir na linguagem e no acesso à memória autobiográfica. Por outro lado, a omissão dos elementos tentativa, conseqüência e reação, podem ter relação com um passado tumultuado e conflitivo, a ponto de tais sujeitos não quererem falar sobre ele, pois

Tabela 3. A linguagem de adolescentes usuários e não usuários de álcool/drogas ${ }^{(16)}$

\begin{tabular}{|c|c|c|c|c|c|c|c|c|}
\hline \multirow[t]{2}{*}{ Elementos } & & \multicolumn{2}{|c|}{ Usuários } & \multicolumn{2}{|c|}{ Não usuários } & \multicolumn{2}{|c|}{ Total } & \multirow[t]{2}{*}{$\mathrm{p}$} \\
\hline & & $\mathrm{N}$ & $\%$ & $\mathrm{~N}$ & $\%$ & $\mathrm{~N}$ & $\%$ & \\
\hline \multirow[t]{3}{*}{ Cenário } & Ausência & 1 & 4,0 & 0 & 0 & 1 & 2,0 & 1,00 \\
\hline & Presença & 24 & 96,0 & 25 & 100,0 & 49 & 98,0 & \\
\hline & Total & 25 & 100,0 & 25 & 100,0 & 50 & 100,0 & \\
\hline \multirow[t]{3}{*}{ Complicação } & Ausência & 0 & 0 & 0 & 0 & & & 1,00 \\
\hline & Presença & 25 & 100,0 & 25 & 100,0 & 50 & 100,0 & \\
\hline & Total & 25 & 100,0 & 25 & 100,0 & 50 & 100,0 & \\
\hline \multirow[t]{3}{*}{ Resposta Interna } & Ausência & 21 & 64,0 & 15 & 60,0 & 31 & 62,0 & 0,115 \\
\hline & Presença & 4 & 36,0 & 10 & 40,0 & 19 & 38,0 & \\
\hline & Total & 25 & 100,0 & 25 & 100,0 & 50 & 100,0 & \\
\hline \multirow[t]{3}{*}{ Tentativa } & Ausência & 11 & 24,0 & 2 & 8,0 & 8 & 16,0 & $0,010^{*}$ \\
\hline & Presença & 14 & 76,0 & 23 & 92,0 & 42 & 84,0 & \\
\hline & Total & 25 & 100,0 & 25 & 100,0 & 50 & 100,0 & \\
\hline \multirow[t]{3}{*}{ Conseqüência } & Ausência & 6 & 16,0 & 0 & 0 & 4 & 8,0 & $0,030^{*}$ \\
\hline & Presença & 19 & 84,0 & 25 & 100,0 & 46 & 92,0 & \\
\hline & Total & 25 & 100,0 & 25 & 100,0 & 50 & 100,0 & \\
\hline \multirow[t]{3}{*}{ Reação } & Ausência & 18 & 64,0 & 9 & 36,0 & 25 & 50,0 & $0,023^{*}$ \\
\hline & Presença & 7 & 36,0 & 16 & 64,0 & 25 & 50,0 & \\
\hline & Total & 25 & 100,0 & 25 & 100,0 & 50 & 100,0 & \\
\hline
\end{tabular}

Teste de Qui-quadrado $\left({ }^{*} \mathrm{p}<0,05\right)$. 
comprometeriam a si próprios e/ou a pessoas com as quais as lembranças tivessem relação, uma vez que a literatura relata estreita relação entre memória autobiográfica, linguagem e emoção ${ }^{(27)}$.

Isoladamente, os elementos menos incidentes (desde ausentes, a pouco presentes) também fornecem informações importantes. Os usuários relatam menos tentativa, ou seja, ao verbalizar suas memórias pessoais, parecem ter menos condições para pensar em estratégias adequadas que permitam resolver o problema vivido, dado consoante com a literatura $^{(28)}$.

O elemento seguinte, conseqüência, também aparece menos incidente na população de usuários, refletindo dificuldades para conectar e expressar verbalmente suas memórias, não desencadeadas em função da ausência ou pouca presença do elemento anterior (tentativa).

Mais uma vez, o fato de os adolescentes usuários de drogas terem relatado menos reação, mostra que, ou houve dificuldades de evocação, ou esta informação foi omitida porque não é positiva e, assim, também não é prazerosa de ser mencionada. Além disso, a ausência deste elemento parece esconder a transgressão e as relações interpessoais comprometidas, deixando transparecer fatos da vida que não devem ser compartilhados.

A análise de uma memória ocorrida na infância de adolescentes usuários de drogas, por meio da linguagem, permite verificar que as lembranças desta população são mais vagas e genéricas. Este dado reflete um possível comprometimento cognitivo (mecanismo de evocação da lembrança) e/ ou de integração da memória à linguagem ${ }^{(29)}$, mas, também, parece fornecer informações acerca de particularidades das histórias de vida desses sujeitos e de sua relação com o uso de drogas.

A maior contribuição deste estudo para a prática fonoaudiológica mostra-se no manejo clínico e na mobilização do adolescente para a abstinência. $\mathrm{O}$ usuário de álcool e drogas tende a negar e a minimizar tanto o uso como as conseqüências deste uso em sua vida. Quando ele entra em contato com as lacunas contidas em sua linguagem oral, começa a questionar-se sobre a forma e o conteúdo de sua comunicação e passa a relacionar a sua fala com o uso de drogas (alterações lingüísticas e cognitivas) e com seu funcionamento psicológico e social, na medida em que se expõe menos ao outro.

Quando o fonoaudiólogo analisa e possibilita a reconstrução de uma linguagem mais estruturada, do ponto de vista lingüístico, o adolescente também inicia um processo de estruturação psíquica, porque passa a refletir sobre o próprio comportamento, o que propicia o auto-conhecimento, habilidade de fundamental relevância para que ele atinja a abstinência e refaça seus laços sociais.

\section{CONCLUSÃO}

O perfil da linguagem representativa da memória autobiográfica de adolescentes usuários de drogas é diferente do de adolescentes não usuários. Este dado, em parte, pode estar relacionado ao comprometimento no funcionamento cognitivo e/ou de linguagem, assim como de aspectos sociais e psíquicos resultantes das vivencias e experiências pessoais e da analise e percepção de mundo. Os dados apontam que estes sujeitos apresentam uma fragmentação da linguagem e da forma como se vêem e entendem suas próprias histórias de vida.

\section{AGRADECIMENTOS}

Ao FUAA (Fundo de Aprimoramento Acadêmico do Instituto de Psiquiatria da Faculdade de Medicina da USP), que financiou a apresentação deste trabalho no "International Congress-World Psychiatry Association” em Istanbul - Turquia, Julho de 2006.

\begin{abstract}
Purpose: To recollect autobiographical memory of adolescent drug users by means of language. Methods: Twenty five male adolescents, alcohol/drugs users and 25 nonusers, with ages between 13 and 17 years, took part on this study. After collection of the verbal speech sample, the analysis was carried out based on the model that determines the discursive structure in setting, complication, internal answer, attempt, consequence and reaction. Results: The group of users produced less attempt $(\mathrm{p}=0,010)$, consequence $(\mathrm{p}=0,030)$ and reaction $(\mathrm{p}=0,023)$, indicating different language profiles between the groups. Conclusion: The autobiographical memory profile of adolescent drug users was different from that of non users. This result can be related to the cognitive and/or linguistic functioning deficit, as well as to damage on social and psychic aspects, causing interruptions in the sequence of recollections.
\end{abstract}

Keywords: Memory; Language; Neuropsychology; Transtornos relacionados ao uso de substâncias; Adolescent 


\section{REFERÊNCIAS}

1. Saxon AJ, Calsyn DA, Haver VM, Delaney CJ. Clinical evaluation and use of urine screening for drug abuse. West J Med. 1988;149(3):296303.

2. Cohler BJ. Personal narrative and life course.In: Baltes PB; Brim Jr OG, editors. Life span development and behavior. Washington, DC.: US Government Printing, 1982. v.4, p. 205-41.

3. Grotevant HD. The integrative nature of identity: Bringing the soloists to sing in the choir. In. Kroger J, editor. Discussions on ego identity. Hillsdale, NJ: Lawrence Erlbaum; 1993. p. 121-46.

4. Rogers RD, Robbins TW. Investigating the neurocognitive deficits associated with chronic drug misuse. Curr Opin Neurobiol. 2001;11(2):250-7.

5. Solowij N, Stephens RS, Roffman RA, Babor T, Kadden R, Miller M, Christiansen K, McRee B, Vendetti J; Marijuana Treatment Project Research Group. Cognitive functioning of long-term heavy cannabis users seeking treatment. JAMA. 2002;287(9):1123-31.

6. Ardila A, Rosselli M, Strumwasser S. Neuropsychological deficits in chronic cocaine abusers. Int J Neurosci. 1991;57(1-2):73-9.

7. Piper BJ, Meyer JS. Memory deficit and reduced anxiety in young adult rats given repeated intermittent MDMA treatment during the periadolescent period. Pharmacol Biochem Behav. 2004;79(4):723-31.

8. White AM. What happened? Alcohol, memory blackouts, and the brain. Alcohol Res Health. 2003;27(2):186-96.

9. Eiber R, Puel M, Schmitt L. [Heroin abuse, autobiographical memory and depression. Encephale]. 1999;25(6):549-57. French.

10. Tulving E, Kapur S, Craik FI, Moscovitch M, Houle S. Hemispheric encoding/retrieval asymmetry in episodic memory: positron emission tomography findings. Proc Natl Acad Sci USA. 1994;91(6):2016-20.

11. Conway MA. A structural model of autobiographical memory. In: Conway MA, Rubin DC, Spinnler H, Wagenaar A, editors. Theoretical perspectives on autobiographical memory. Dordrecht: Kluwer Academic; 1992. p. 167-94.

12. Matsumoto A, Stanny CJ. Language-dependent access to autobiographical memory in Japanese-English bilinguals and US monolinguals. Memory. 2006;14(3):378-90.

13. Radvansky GA, Copeland DE, Zwaan RA. A novel study: investigating the structure of narrative and autobiographical memories. Memory. 2005;13(8):796-814.

14. Stein N, Glenn C. An analysis of story comprehension in elementary school children. In: Freedle RO, editor. New directions in discourse processing. Norwood: Ablex; c1979. [Advances in Discourse Processes, 2].

15. Habermas T, Bluck S. Getting a life: the emergence of the life story in adolescence. Psychol Bull. 2000;126(5):748-69.
16. Peterson C, McCabe A. Development psycholinguistics: three ways of looking at a child's narrative. New York: Plenum; c1983.

17. Blagov PS, Singer JA. Four dimensions of self-defining memories (specificity, meaning, content, and affect) and their relationships to selfrestraint, distress, and repressive defensiveness. J Pers. 2004;72(3):481511.

18. Diagnostic and statistical manual of mental disorders: DSM-IV. 4a ed. Washington: American Psychiatric Association; 1994.

19. Almeida PM, Wickerhauser H. O critério ABA-ABIPEME: em busca de uma solução. Um estudo e uma proposta submetidos à ABA Associação Brasileira de Anunciantes e ABIPEME - Associação Brasileira dos Institutos de Pesquisa de Mercado. São Paulo; 1991.

20. Substance Abuse and Mental Health Services Administration. Office of Applied Studies (1998). National Household Survey on Drug Abuse. Rockville, MD.: U.S. Department of Health and Human Services; 1999.

21. Substance Abuse and Mental Health Services Administration. Office of Applied Studies (1996). Substance Abuse in States and Metropolitan Areas: Model Based Estimates from the 1991-1993, National Household Survey on Drug Abuse: Summary Report. Rockville, MD.: U.S. Department of Health and Human Services; 1996.

22. Carlini EA, Galduroz JCF, Noto AS, Nappo SA. I Levantamento domiciliar sobre o uso de drogas psicotrópicas no Brasil: estudo envolvendo as 107 maiores cidades do país - 2001. São Paulo: CEBRID/ UNIFESP; 2002.

23. Haddad N. Noções gerais de amostragem. In: Haddad N. Metodologia de estudos em ciências da saúde: como planejar, analisar e apresentar um trabalho científico. São Paulo: Roca; c2004.

24. Erikson EH. Identity, youth, and crisis. New York: W. W. Norton; c1968.

25. McGue M, Iacono WG. The association of early adolescent problem behavior with adult psychopathology. Am J Psychiatry. 2005;162(6):1118-24.

26. Catal LL, Fitzgerald JM. Autobiographical memory in two older adults over a twenty-year retention interval. Mem Cognit. 2004;32(2):311-23.

27. Schaefer A, Philippot P. Selective effects of emotion on the phenomenal characteristics of autobiographical memories. Memory. 2005;13(2):14860.

28. Bechara A, Dolan S, Denburg N, Hindes A, Anderson SW, Nathan PE. Decision-making deficits, linked to a dysfunctional ventromedial prefrontal cortex, revealed in alcohol and stimulant abusers. Neuropsychologia. 2001;39(4):376-89.

29. Ceballos NA, Houston RJ, Smith ND, Bauer LO, Taylor RE. N400 as an index of semantic expectancies: differential effects of alcohol and cocaine dependence. Prog Neuropsychopharmacol Biol Psychiatry. 2005;29(6):936-43. 